\title{
Integrated Train Rescheduling and Rerouting during Multidisturbances under a Quasi-Moving Block System
}

\author{
Peijuan Xu $\mathbb{D}$, ${ }^{1}$ Dawei Zhang $\mathbb{D},{ }^{2,3}$ Jingwei Guo $\mathbb{D}^{\mathbb{D}},{ }^{4}$ Dan Liu $\mathbb{D}$, ${ }^{1}$ and Hui Peng $\mathbb{D}^{1}$ \\ ${ }^{1}$ School of Transportation Engineering, Chang'an University, Xi'an 710064, China \\ ${ }^{2}$ School of Automobile, Chang'an University, Xi'an 710064, China \\ ${ }^{3}$ State Key Laboratory of Traction Power, Southwest Jiaotong University, Chengdu 610031, China \\ ${ }^{4}$ School of Energy Science and Engineering, Henan Ploytechnic University, Jiaozuo 454003, China \\ Correspondence should be addressed to Dawei Zhang; dwzhang@chd.edu.cn
}

Received 18 November 2020; Revised 24 February 2021; Accepted 18 March 2021; Published 8 April 2021

Academic Editor: Keping Li

Copyright $\odot 2021$ Peijuan Xu et al. This is an open access article distributed under the Creative Commons Attribution License, which permits unrestricted use, distribution, and reproduction in any medium, provided the original work is properly cited.

\begin{abstract}
It is known that it is critical for train rescheduling problem to address some uncertain disturbances to keep the normal condition of railway traffic. This paper is keen on a mathematical model to reschedule high-speed trains controlled by the quasi-moving blocking signalling system impacted by multidisturbances (i.e., primary delay, speed limitation, and siding line blockage). To be specific, a mixed-integer linear programming is formulated based on an improved alternative graph theory, by the means of rerouting, reordering, retiming, and train control. In order to adjust the train speed and find the best routes for trains, the set of alternative arcs and alternative arrival/departure paths are considered in the constraints, respectively. Due to this complex NP-hard problem, a two-step algorithm with three scheduling rules based on a commercial optimizer is applied to solve the problem efficiently in a real-word case, and the efficiency, validity, and feasibility of this method are demonstrated by a series of experimental tests. Finally, the graphical timetables rescheduled are analysed in terms of free conflicts of the solution. Consequently, the proposed mathematical model enriches the existing theory about train rescheduling, and it can also assist train dispatchers to figure out disturbances efficiently.
\end{abstract}

\section{Introduction}

High-speed railway faced a rapid development in the world and showed competitive advantages in the passenger market, due to its better performance in safety, fast speed, and effectiveness. However, some inevitable disruptions deviate actual train running lines from its original planned lines in time/space horizons. As delay time degenerates satisfaction of passengers to some degree, it is essential to explore and carry out some optimal solutions/measures to recovery normal conditions of railway traffic.

In recent years, train rescheduling problem is one of hot research topics in railway operation and management. According to the state-of-art research outcomes, retiming, rerouting, reordering, and cancelling trains are mainly four kinds of measures [1], which are used to mitigate the negative influences resulted from disturbances or disruptions for the railway system. Furthermore, certain articles focused on the self-learning decision technology [2] or decision support system [3] to enhance the robustness of timetables by the means of iteratively evaluation from subsequent time horizons or the so-called offline railway traffic dispatching.

Those strategies usually aim at minimizing operation conflicts and train delays for a single/double-tracked railway line/network area on operational conditions and accident characteristics [4]. However, train rescheduling and rerouting are often applied to a multistep formulation or multi-iterations, due to the complexity of this problem [5]. In order to solve the negative influence of disruptions/ disturbances effectively, mathematical formulation is proved to be one of common methods by describing railway operation as a series of constraints, e.g., integer programming (IP) [6] model, mixed-integer programming (MIP) model 
[7], and alternative graph (AG) model [8]. From the aspect of different granularities of railway infrastructure or trains activities, current existed optimal mathematical programming can be divided into two groups, i.e., macroscopic model and microscopic model. It is common that the microscopic models are applied on a (part of) independent railway line impacted by disturbances or minor disruptions. On the other hands, the macroscopic is able to solve a wider railway area, e.g., a regional network, multitracked area, and a comprehensive central junction impacted by disruptions [9]. The macroscopic model treats railway stations and segments (i.e., the track between two consecutive railway stations) as basic independent units. However, the microscopic model takes signals along the rail track, switches, and block sections fully into consideration [10]. The macroscopic model is good at addressing some serious disruptions $[11,12]$, but it may ignore some conflicts among trains or infrastructure, whose solution cannot be used for practical dispatching work directly.

Concerning this defect and characteristics of the quasimoving block signalling system, herewith, we mainly focus on applications related to microscopic models.

Alternative graph, a kind of microscopic model, is widely used to deal with the influence caused by mirror/slight disruptions/disturbances in a railway system. In the model, occupation relations between trains and block sections are seen as a job-shop scheduling problem, which can be figured out by alternative graph [13]. When alternative graph theory adopts rerouting in railway traffic management, some heuristic algorithms, i.e., tube search [14] or neighborhood search [15], are usually designed to overcome the HP hard problems. Furthermore, a variety of objective functions have been studied and analysed, which include the minimization of secondary (consecutive) delay and final delay (tardiness), combined with sophisticated multiobjective optimization framework in [16]. Meanwhile, a practicality and validity of alternative graph for reducing the delays are tested. Besides, most of those applications of alternative graph focus on the fixed-blocking system. As most as we know, few papers studied the moving blocking system [17] and quasi-moving block system [18]. Xu et al. [18] applied a set of alternative arcs to illustrate the characteristics of the quasi-moving block system in the Chinese high-speed railway system, but the rerouting was not involved in the model. Furthermore, the smooth of train speed curves is optimized by train control and traffic management at one iteration [19]. From the aspect of high-speed train rescheduling problem, the detailed train speed trajectories are considered [20], where only 2 trains operating on a part of railway line with two stations effected by speed restriction are studied in the experimental test. On the other hand, an MIP model based on a stochastic optimization method is established to handle disturbance on a high-speed railway [21]. Nevertheless, the microscopic model is subject to some defects in dealing with some serious disruptions in the railway system. In order to improve the generalization and robustness of real-time Chinese high-speed train rescheduling methods, it is essential to consider rerouting, once some track section is blockage.
Concerning above analysis, a mixed-integer linear programming (MILP) model based on an improved alternative graph related to Chinese high-speed railway traffic is established to enrich real-time train rescheduling methods by involving rerouting, retiming, and reordering simultaneously in this paper. The model complements the optimization model proposed by $\mathrm{Xu}$ et al. [18] which focuses on train rescheduling under speed restriction and neglects train rerouting. This paper makes the following contributions:

(i) A mixed-integer linear programming (MILP) model is established to solve the real-time rescheduling problem for the Chinese high-speed railway traffic, under the combined influence of primary delay, the disruptions related to speed limitation, and the blockage of siding lines. The combination of disturbances is common in realworld railway operation activates and is studied seldom for its complexity, as most as we know.

(ii) We improved the general job-shop model to achieve train retiming, rerouting, and reordering, and speed management simultaneously, where the constraints related to alternative paths in railway stations are erected according to the "Big- $\mathrm{M}^{\prime \prime}$ principle. Furthermore, the train entrance path and departure path in railway stations area are computed from the view of rail track circuit.

(iii) Due to the difficulty of model, a two-step procedure with three kinds of scheduling rules are applied, such as space headway, headway time interval, and free-conflict rule. In the real-world high-speed railway experiments, it is proved that this solving method can figure out the solution with good quality in an acceptable computation time.

The detail content of this paper is organized as follows. Section 2 explains the disruptions studied and the basic methodology, and the proposed mathematical model improved is built to minimize delay times and times of changing routes in Section 3. Furthermore, the experimental test, solving method, and the result analysis are given in Section 4. Finally, conclusions and directions for future research are reported in Section 5.

\section{Problem Description and Alternative Graph Theory}

2.1. Problem Description. Some inevitable perturbations, which are caused by some infrastructure failures, bad weather condition, or human errors, cause conflicts between trains or infrastructure. The abnormal condition is unable to meet the requirement of trains in full-speed, thus lower running speed in the area impacted is executed, or rerouting/ detour is applied to avoid the broken track area. In this paper, disturbances studied refer to the combination of primary delay, blockage of siding line (s), and speed limitation (s) in segment (s), illustrated in Figure 1:

(1) Primary delay: before trains enter the given railway line, some delay times of trains were generated in 


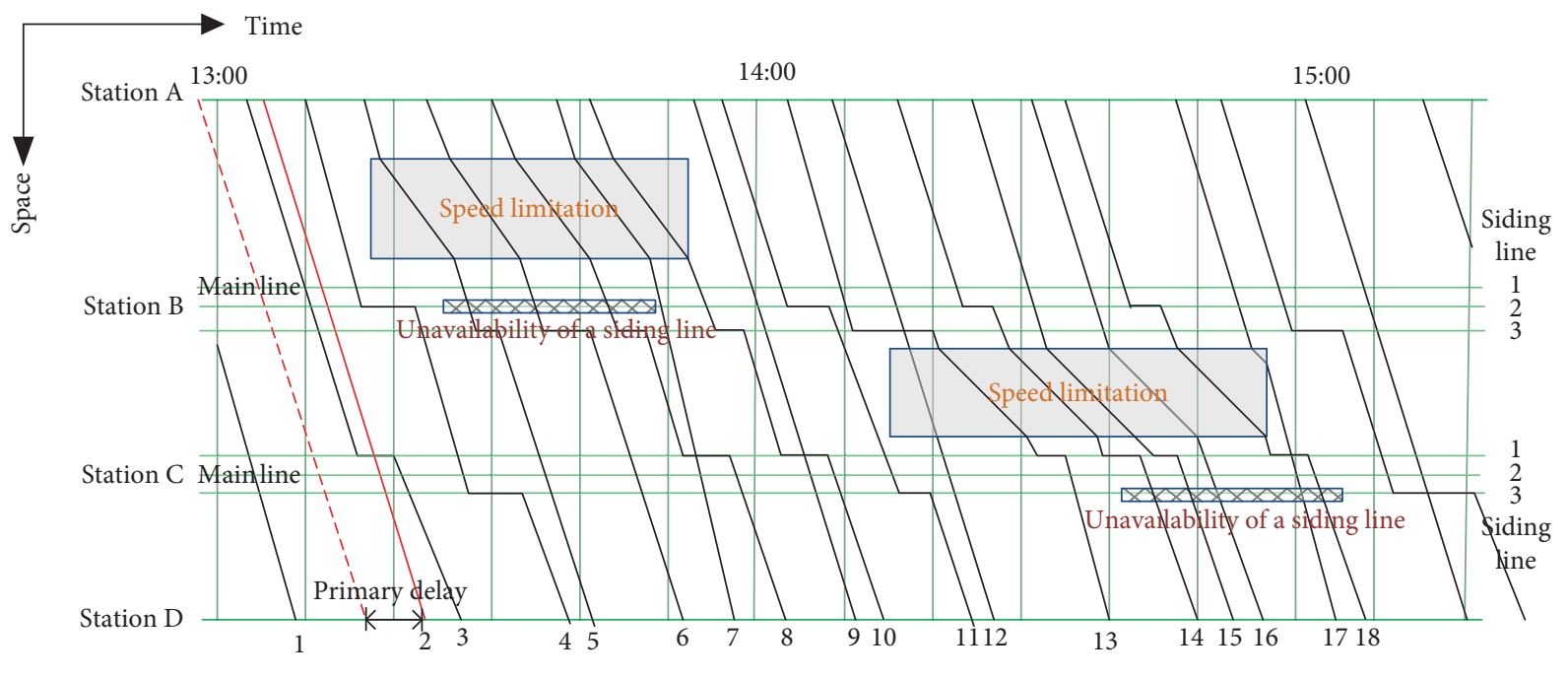

FIGURE 1: Illustration for multidisturbances.

their previous routes. In Figure 1, the actual running line of train no.2 (train numbers are listed at the bottom of Figure 1) switches from the red dash line to the red solid line, due to the input of primary delay.

(2) Disturbances related to speed limitation: in high-speed railway system, once bad weather condition (i.e., heavy rain/snow, strong wind, and fog), switch/signalling failures, and any other infrastructure failures occur, one of common efficient response actions is to force high-speed trains to reduce their speeds in the impacted area. Therefore, extra running time is generated by the speed limitation. The speed limitations studied can vary from different time spans, geographical spans, and different speed levels in this paper. There are two speed limitation cases shown by the shaded rectangles in Figure 1, where trains nos. $5,6,7,8,10,13,14,15,16,17$, and 18 are impacted.

(3) Blockage of siding line in railway station: sometimes siding line in a railway station is blocked by infrastructure failures, maintenance activities, or passenger activities, so all trains cannot dwell on the impacted line for passenger alighting or boarding activities. Thus, the original plan for the usage of the impacted siding line should be adjusted to avoid potential conflicts or accidents, as well as their entrance path and departure path. As shown in Figure 1 , when the siding lines blocked in station $\mathrm{B}$ (siding line 2) and station $C$ (siding line 3 ), respectively, and trains nos. 5, 6, and 7 or train nos. 14, 15 , and 18, which are planned to dwell in blockage of siding lines, have to change their siding lines in advance. Undoubtedly, making new arrival path and departure path are essential to adjust the siding line.

As mentioned above, the disturbances studied in this paper are a complicated combination of primary delay, speed limitation, and blockage of siding line. They deteriorate the solving process of the problem and increase the probability of occurrence of conflicts between trains.

\subsection{Alternative Graph Theory and Job-Shop Scheduling} Problem. In general, train operation can be seen as a jobshop scheduling problem, and the occupation relationship between the train and the block section is similar with the inprocess of a good in a machine. In this way, high-speed rail line can be divided into many sections according to the signals or track circuits, and each section can only be utilized at most one train at the same time. When two more trains apply for one section simultaneously, there is a sequence decision process to decide which train has the priority to use the section. The latter train can only use the section after it is released by the former train. As shown in the top of Figure 2, there are three trains of the same direction going to pass a railway station and the scheduled paths of trains $t_{1}, t_{2}$, and $t_{3}$ are 1-2-3-6-7-8-9, 1-2-3-5-7-8-9, and 1-2-4-8-9, respectively. Thus, train $t_{1}$ and train $t_{2}$ are designed to stop and dwell at siding in Sections 6 and 5, respectively. Train $t_{3}$ is a passing-through train by main line (Section 4). Furthermore, the relationship between trains and railway infrastructure is represented in the bottom of Figure 2, which manages to transfer the railway operation activities into an alternative graph to optimize occupation sequence of trains. To be specific, the node presents railway block section, and the outgoing arc defines the movement of one train between two neighboring block sections, and the operation time of each movement (/arc) refers to the running time/entrance time/departure time/headway time of the outgoing section, which is the weight of each arc. In terms of arcs, the solid arc is called fixed arc, which can determine train routes according original plan, and the red dash arcs are the entrance deifications for trains, whose weights refer entrance times of trains scheduled by the timetable while the planned departure times are depicted by green dash arcs which require that trains cannot leave the station until the departure time. Last but not least, the pair of dark dash lines with a red 


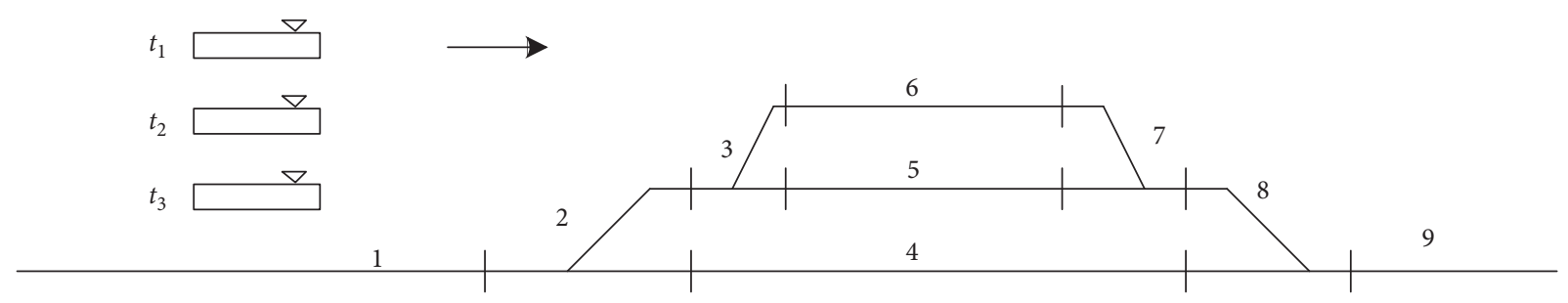

(a)

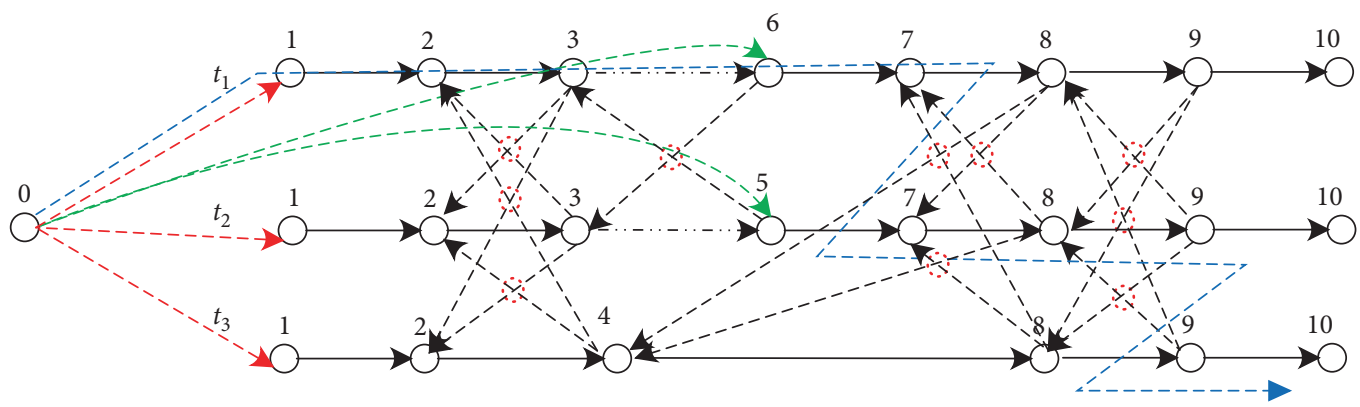

(b)

FIgURE 2: Alternative graph with two trains in one railway station.

circle at their crossing defines a pair of alternative arcs, which is used to determine the train order for the usage priority to the same rail section. In detail, for the potential conflicts caused by the same section occupation between different trains (such as Section 2 and Section 3 for those three trains in Figure 2), there are three pairs of alternative arcs which are designed to avoid potential collision between different trains. Considering the requirements of switches, sections, or signals, the headway time between two consecutive trains is treated as the weight of each pair of alternative arcs. Consequently, the headway time of alternative arcs $((2,3),(2,4))$ and $((8,9),(8,9))$ values to required headway time. In terms of the best solution, the minimum of maximum time from entry node to exit node is the critical route (the blue dash line) to get the better solution with more capacity conservation.

In respect of the requirement of the quasi-moving block system, a new space interval restrict is as well taken into account to keep safety distance for two continue trains in open track area. In particular, when a train wants to enter a given section with a certain speed, there must be enough empty sections corresponding to speed level in front of this train to keep safety in case of emergency braking. A series pairs of alternative arcs of each train were generated to demonstrate different speed levels for the train on a section [18]. For instance in Figure 3, the red, purple, origin, blue, and green dash lines represent speed level 1, 2, 3, 4, and 5, respectively, and the bigger the speed level is, the more the free section in front required. Obviously, this kind of alternative arcs exists in the segment between two consecutive trains.

Based on the alternative graph theory, all of constraints related to train operations are transformed into a network graph, and all the arcs in the network graph can be represented as mathematical constraints. The detailed formulation is shown as follows:

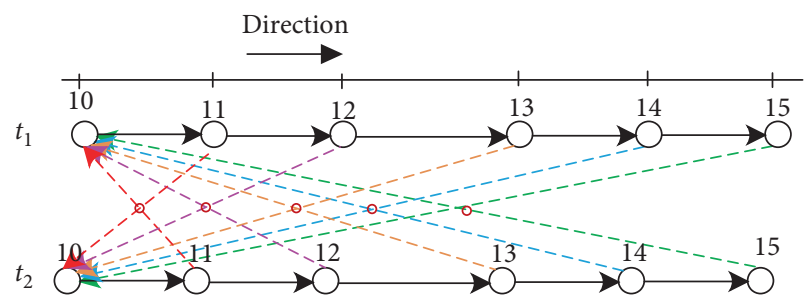

FIgURE 3: The alternative arcs for headway interval.

objective: $\min : \omega_{n}-\omega_{0}$,

$$
\omega_{i, q}-\omega_{i, p} \geq f_{p q}, \quad((p, q) \in F)
$$

$$
\left(\omega_{j, q}-\omega_{i, p} \geq \delta_{p q}\right) \vee\left(\omega_{i, k}-\omega_{j, h} \geq \delta_{h k}\right)((p, q),(h, k) \in A),
$$

$\omega_{j, q}-\omega_{i, p} \geq 0 \vee \omega_{i, q}-\omega_{j, p} \geq 0, \quad\left(p \geq q+\xi, \beta_{\xi, \text { LaterTrain }, q}=1\right)$.

Constraints (4) can be transferred into the following two constraints:

$$
\begin{aligned}
& \omega_{j, q}-\omega_{i, p} \geq-\left(2-\mu_{i, j}-\beta_{\xi, j, q}\right) M, \\
& \omega_{i, q}-\omega_{j, p} \geq-\left(1+\mu_{i, j}-\beta_{\xi, i, q}\right) M,
\end{aligned}
$$

where $\forall i, j \in T, \xi \in B p, q \in \in S: i \neq j, q=p+\xi$.

The ambition of this model is to reduce traffic tardiness, shown by equation (1), where $\omega_{n}$ and $\omega_{0}$ denote the arrival time of the last train at the end node and the start time of the first train at the original, respectively. In the final solution, each train finds a path, which consists of nodes and arcs, and the minimum of the longest path is the critical path, which 
determines the quality of rescheduled timetable directly. Constraints (2) relate to fixed arcs, which is used to control the running time/arrival time/departure time for each train on each section (the fixed arc), where, $f_{p, q}$ denotes the minimal running time to pass through one section, and $\omega_{i, q}$ is the start time when train $i$ utilizes block section $q . F$ is the set of fixed arcs, and $(p, q)$ represents a fixed arc. In this way, the requirements of trains' entrances of the original, primary delays, and departure times can be formulated by those constraints. On the other hand, alternative arcs are described by constraints (3), where $\delta_{p q}$ and $\delta_{h k}$ represent minimal headway time interval to keep trains use the sections of potential conflicts separately. It should be noted that $A$ is the set of alternative arcs and $((p, q)$ and $(h, k))$ is a pair of alternative arcs. Furthermore, there is a disjunctive relationship in constraints (3); in other words, only one subconstraint is tight constraint during solving process. Considering the features of train speed and their safety braking distance, constraints (4) are used to deal with those alternative arcs (i.e., $\operatorname{arc}(10,11),(10,12),(10,13),(10,14)$, and $(10,15)$ in Figure 3$)$, with speed levels in the open track area. To be specific, the distance headway to be kept free between two consecutive trains depends dynamically on the speed level of the following train. We thus require that at least $\xi$ block sections should be kept free between two consecutive trains, when the second train runs at the speed level $\xi\left(\beta_{\xi, \text { LaterTrain,q }}=1\right) . \beta_{\xi, \text { LaterTrain,q }}$ denotes that the later/ second train runs on section $q$ with speed level $\xi$. With the involvement of the big- $\mathrm{M}$ structure, constraints (5) and (6) are implemented in the actual mathematically model, where $\mu_{i, j}$ represents the order of train $i$ and $j$ in the open track area, and if $\mu_{i, j}=1$, train $i$ is in front of train $j$; otherwise, $\mu_{i, j}=0$. In this way, the basic model can be applied to reschedule trains in a quasi-moving block signalling system.

\section{High-Speed Train Rescheduling Model Integrating Rerouting}

The basic alternative graph can be applied to train rescheduling with a quasi-moving block signaling system. The influence of multidisturbances makes it harder to reorder, reroute, and retime trains efficiently, due to the complex combinatorial problems. According to the stateof-art outcomes related to train rescheduling, there are few models which can solve multidisturbances and train speed control simultaneously. Concerning actual demanding of high-speed railway operation, an MILP model based on the alternative graph is proposed in this paper.

3.1. Assumption and Decision Variables. First of all, some notions in this model are illustrated in Figure 4. And it should be noted that the meaning of rerouting in this paper refers to choose alternative siding lines and arrival/departure paths in one/one more railway station area for the impacted trains. Before formulation, we made assumptions as follows:
(1) The given disturbances cannot result in cancelation of train (s) in any time.

(2) Parameters of speed limitations and blockages of siding lines are known once they happen.

(3) Passing-through trains are forbidden to change their routes in stations. Only those trains which are scheduled to stop can change their routes in the planned stop stations.

The parameters of trains operation, railways lines, and technical activities are described as follows: $t, i, j(t, i, j \in T)$ denote indexes of trains, and $T$ is the set of trains. $s, p, q(s, p$, $q \in S$ ) denote indexes of block sections, and $S$ is the set of block sections. $A$ and $F$ denote sets of alternative arcs and fixed arcs, respectively. $\xi$ denotes the speed levels of the train, i.e., 1, 2, 3, 4, and 5, and the bigger the value is, the faster the train runs. $y$ is the index of speed limitation case, and $M$ is a big enough integer. The decision variables in the model are shown as follows:

$\omega_{t, s}$ : the start time when train $t$ utilizes block section $s$.

$z_{t, s}$ : binary decision variable, i.e., if $z_{t, s}=1$, train $t$ uses section $s$; otherwise, section $s$ is not used. Section $s$ is among

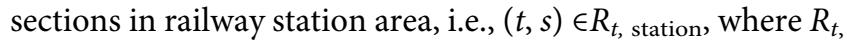
station is the set of block sections in station area which can be used by train $t$.

$\lambda_{i, j, p, q}$ : binary variable which describes the sequence between a pair of trains $(i, j)$ for occupations of alternative $\operatorname{arc}(p, q)$. If $\lambda_{i, j, p, q}=1$, the time when train $i$ uses section $p$ is earlier than that of train $j$ or vice versa.

$\beta_{\xi, t, s}$ : binary variable which represents whether train $t$ runs on section $s$ with the $\xi$ th speed level. If $\beta_{\xi, t, s}=1$, train $t$ runs on section $s$ with $\xi$ th speed level, or vice versa.

$x_{t, s, y}$ : binary variable which demonstrates whether the start time $\omega_{t, s}$ is later than the start time of the $y$ th speed limitation in impacted section $s\left(s \in \overline{S_{y}}\right)$. If $x_{t, s, y}=1$, $\omega_{t, s} \leq T_{1 y}$, or vice versa.

$\overline{x_{t, s, y}}:$ binary variable which demonstrates whether the start time $\omega_{t, s}$ is earlier than the end time of the $y$ th speed limitation in impacted section $s\left(s \in \overline{S_{y}}\right)$. If $x_{t}, s, y=1$, $\omega_{t, s} \geq T_{1 y}$, or vice versa.

$\operatorname{dev}_{t, s}$ : binary variable which represents absolute deviation between $z_{t, s}$ and original planned $z_{t, q}^{0}$ (which is the original usage plan of section $q$ in normal condition). If $\operatorname{dev}_{t, s}=1\left((t, s) \in R_{t, \text { station }}\right)$, train $t$ changes its usage plan for section $s$, or vice versa.

$d e_{t}$ : summation of $\operatorname{dev}_{t, s}$ for train $t$.

3.2. Model Formulation concerning Siding Line Resetting. In normal condition, the arrival and departure paths in railway stations are almost fixed once the original timetable given. In this way, passengers and railway staff can wait for the train or arrange routes for the trains in advance. However, due to the influence of disturbances, some unavailable routes have to be changed to reduce tardiness in the traffic. In order to avoid inconvenience caused by changing train routes frequently, to minimize total times of changing siding lines is considered in the objective of this model, as 


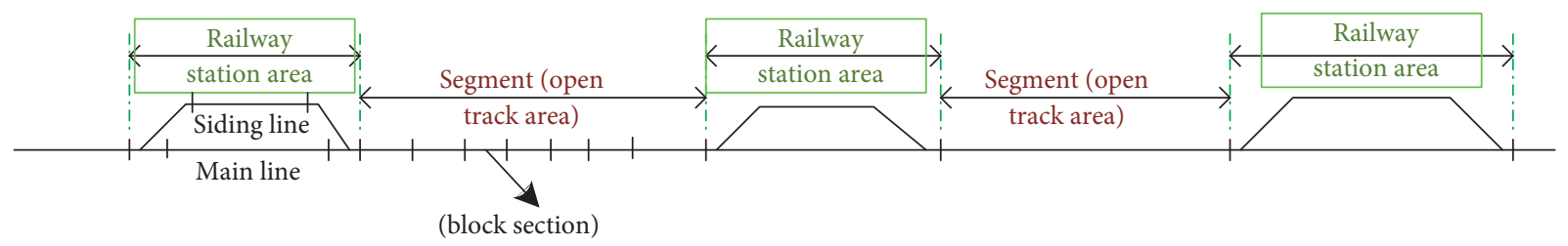

FIgURE 4: Graphical illustration of notions related to railway track.

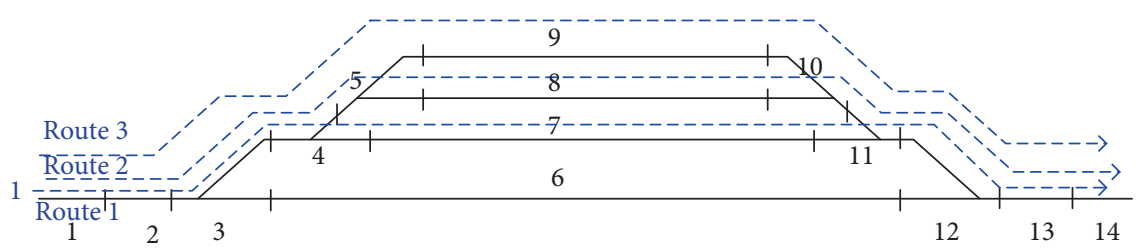

(a)

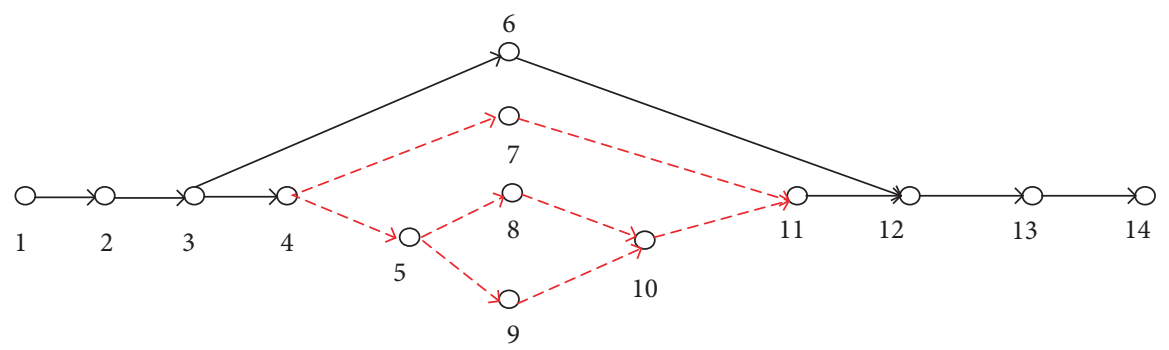

(b)

FIgURE 5: Alternative routes for one train in a railway station area.

well as tardiness of trains. The proposed objective is listed as follows:

$$
\text { objective: } \min : \sum_{t \in T}\left(\omega_{t, \text { end }}-e_{t}-c_{t}-d t_{t}\right)+\alpha \sum_{t \in T} \mathrm{de}_{t} \text {, }
$$

where $\omega_{t, \text { end }}$ denotes actual arrival time of train $t$ in the end of given railway track, and $e_{t}, c_{t}$, and $\mathrm{dt}_{t}$ are entrance time, planned travelling time, and primary time of train $t$, respectively, which are known in advance. The first summation in equation (7) denotes the tardiness of traffic, and the second summation represents the times of changing routes. Furthermore, in order to balance the delay time and times of changing routes, the weight $\alpha$ is considered in the following research.

3.2.1. Constraints Related to Rerouting. Once the siding line is blocked, setting alternative routes for impacted trains is essential to keep their routes available. Illustrated by Figure 5, there are 3 alternative paths (i.e., paths 1, 2, and 3, denoted by blue dash lines) for trains to stop, and the corresponding alternative graph is in the bottom half of Figure 5. As the uncertainty of paths leads the uncertainty of potential conflicts, we set constraints as the following two groups:

$$
\begin{aligned}
\omega_{j, p}-\omega_{i, q} \geq & \delta_{p, q}-\left(2-\lambda_{i, j, p, q}-z_{i, q}\right) M, \\
& \forall i, j \in T,(p, q) \in A, q \in R_{i, \text { station }}, p \in R_{j}, i \neq j,
\end{aligned}
$$

$$
\begin{aligned}
\omega_{i, p}-\omega_{j, q} \geq & \delta_{p, q}-\left(1+\lambda_{i, j, p, q}-z_{j, q}\right) M, \\
& \forall i, j \in T,(p, q) \in A, q \in R_{j, \text { station }}, p \in R_{i}, i \neq j,
\end{aligned}
$$

where $\delta_{p, q}$ denotes the minimal headway time between trains $i$ and $j$.

Those two constraints are used to determine the first section among alternative paths. In the other words, the start of arc is certain and the end of arc is uncertain. Constraints (8) and (9) are generally applied in arrival paths with 


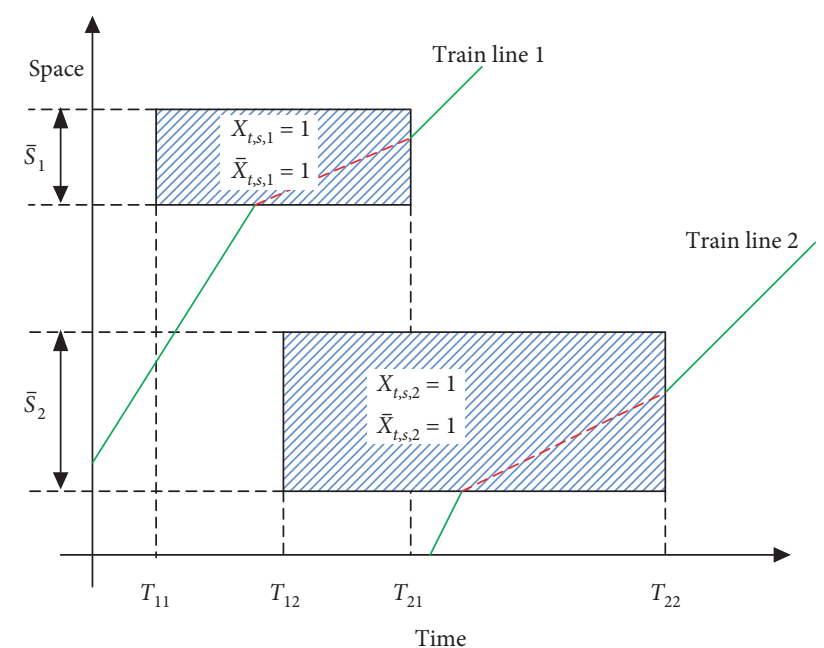

Figure 6: Identification method for multidisturbances related to speed limitation.

switches, joints, or siding lines. Specially, section $q$ is alternative in the $\operatorname{arc}(p, q)$, e.g., section 5 in the $\operatorname{arc}(4,5)$ or section 7 in the arc $(4,7)$ in Figure 5. Furthermore, for those arcs with two uncertain nodes, the relative constraints are built as follows:

$$
\begin{aligned}
\omega_{j, p}-\omega_{i, q} \geq & \delta_{p, q}-\left(3-\lambda_{i, j, p, q}-z_{j, p}-z_{i, p}\right) M, \quad \forall i, j \in T, \\
& (p, q) \in A, p \in R_{i, \text { station }} \cap R_{j, \text { station }}, i \neq j, \\
\omega_{i, p}-\omega_{j, q} \geq & \delta_{p, q}-\left(2+\lambda_{i, j, p, q}-z_{i, p}-z_{j, p}\right) M, \\
& \forall i, j \in T,(p, q) \in A, p \in R \cap R_{j, \text { station }}, i \neq j .
\end{aligned}
$$

Due to uncertainty of former section (which is mentioned in constraints (8) and (9)) of one arc, two route-selection variables, $z_{j, p}$ and $z_{i, p}$, are added in constraints (10) and (11). Those conflicts researched are possible to appear, only when two trains choose the same route in station area. For example, if the following train wants to utilize section 8 in Figure 5, only trains which apply for section 8 indeed are involved by above constraints, and only those trains can cause potential conflicts in section 8. To sum up, those two constraints mainly exist in arcs related to siding lines or departure paths.

In this way, constraints (3) (4) and (8) (11) are able to cover all possible conflicts resulting from the utilization application for the same section or its conflicting section (s).

3.2.2. Constraints about Train Technical Activates. Due to the uncertainty of train route, the running times for the impacted trains have to be changed according to the selected sections among their final routes. As those sections are mainly in the railway station area, the constraints related to the running times refer to the requirement of departure time and dwell time:

$$
\begin{aligned}
\omega_{t, q}-\omega_{t, p}+\left(1-Z_{t, p}\right) M \geq & f_{p}+d_{t, p}, \\
& \forall t \in T, q \in R_{t}, p \in D_{t}, \\
\omega_{t, q}-\omega_{t, p}+\left(1-Z_{t, q}\right) M \geq & f_{p}, \\
& \forall t \in T, p, q \in S, p \in R_{t}, \quad \\
& (p, q) \in F, q \in R_{t, \text { station }} \\
\omega_{t, q}-\omega_{t, p}+\left(1-Z_{t, p}\right) M \geq & f_{p}, \quad \forall t \in T, p, q \in S, q \in R_{t}, \\
& (p, q) \in F, p \in R_{t, \text { station }},
\end{aligned}
$$

where $f_{p}$ is the minimal running time of section $p$; $d_{t, p}$ is the dwell time of train $t$ on section $p$. In detail, constraints (12) represent requirements of dwell times for the potential changeable siding lines. Constraints (13) and (14) deal with running times for uncertain sections selected in arrival/ departure paths, respectively.

3.2.3. Constraints about Disturbances. Regarding to multidisturbances, the following constraints take them into consideration. In detail, the primary delay is considered by constraints (15), where $d t_{t}$ is the primary delay time of train $t$. Constraints (16)-(18) relate to disturbances about speed limitations, where $T_{1 y}$ and $T_{2 y}$ denote the start time and end time of the $y$ th speed limitation, respectively, and $\overline{S_{y}}$ is the set of sections impacted by $y$ th speed limitation, and $\overline{B_{m, y}}$ refers to the maximum speed impacted by the $y$ th speed limitation. There are two speed limitation cases in Figure 6, where the relative parameters and variables are displayed. To the best of our knowledge, almost no research concerns several speed limitations in one train rescheduling case. In this paper, different combinations of speed limitations can be formulated by this model. The variables $x_{t, s, y}$ and $\overline{x_{t, s, y}}$ are used to identify the impacted trains precisely with respect to geospatial direction and time direction. As shown in Figure 6 , once the train running line passes through the rectangle, the trains are impacted and the overlapped part (the red dotted line) indicates the scope of influence. At the same time, those two variables both value to 1 at the same time. In this way, $\overline{B_{m, y}}$ in constraints (18) is a contributing factor:

$$
\begin{aligned}
& \omega_{t, s}-t_{0} \geq e_{t, s}+d t_{t}, \quad \forall t \in T,(t, s) \in E_{T}, \\
& x_{t, s, y} M \geq \omega_{t, s}-T_{1 y}, \quad \forall t \in T, s \in \overline{S_{y}} \cap R_{t}, y \in[1, n u],
\end{aligned}
$$

$$
\overline{x_{t, s, y}} M \geq T_{2 y}-\omega_{t, s}, \quad \forall t \in T, s \in \overline{S_{y}} \cap R_{t}, y \in[1, n u],
$$

$$
\begin{aligned}
\sum_{\xi=1}^{\overline{B_{m, y}}} \beta_{\xi, t, s} \geq & x_{t, s, y}+\overline{x_{t, s, y}}-1, \\
& \forall t \in T, s \in \overline{S_{y}}, s \in R_{t}, \xi \in \mathrm{B}, y \in[1, n u] .
\end{aligned}
$$


Furthermore, constraints about siding line blockage are illustrated by the following constraints:

$$
\begin{aligned}
z_{t, p} & =0, \quad \forall t \in T, p \in \overline{R_{t, \text { station }}}, \\
\omega_{t, q} & \leq z_{t, q} M, \quad \forall t \in T, q \in S_{r}, \quad(t, q) \in R_{t, \text { station }}, \\
\sum_{s \in S_{r}} z_{t, s} & =1, \quad \forall t \in T, s \in S_{r}, r=1,2,3 \ldots . \\
\operatorname{dev}_{t, q} & \leq z_{t, q}^{0}-z_{t, q}, \quad \forall t \in T, q \in R_{t, \text { station }}, \\
\operatorname{dev}_{t, q} & \geq z_{t, q}^{0}-z_{t, q}, \quad \forall t \in T, q \in R_{t, \text { station }} \\
\operatorname{de}_{t} & =\sum_{p \in S_{r}} \operatorname{dev}_{t, p}, \quad \forall t \in T, p \in S_{r} .
\end{aligned}
$$

Constraints (19) require that all trains cannot stop on blocked section $p$ in the set of blockage sections $\left(\overline{R_{t, \text { station }}}\right)$. Constraints (20) set the start times of those alternative sections unselected by train $t$ to 0 . Only one route in one railway station area is chosen for each train, so constraints (21) require that the sum of $z_{t, p}$ related to all siding lines and main lines is equal to 1 . Constraints (22) and (23) combine to measure times of changing sections, compared to the original planned route. In this way, the value of $\operatorname{dev}_{t, q}$ is always a nonpositive number, which is described by constraints (24).

In addition to above constraints, train control related to speed adjustment is still taken into consideration. Those relative constraints refer to constraints (5) (6) in the introduction part for the alternative graph.

\section{Computational Experiments}

4.1. Two-Step Solution Approach with Different Solving Strategies. Train rescheduling problem is demonstrated to be one of hardest combinatorial optimization problems (D'Ariano et al. 2007), and computational times mainly depend on the granularities of formulations. As the proposed model is a microscope model, the amount of constraints and variables reaches easily very large numbers: the number of variables related to speed grows with a function $5 \mathrm{~nm}$ being $n$ the amount of trains and $m$ the amount of sections. Secondly, the constraints related to distance headway (speed selection) of each pair of trains play an even larger role, increasing with $5 n^{2} m-5 m$. Furthermore, the alternative routes of trains make the model more complicated, so the raw amount of constraints related to route selection increasing with $n p$ ( $n p-1)$, where $p$ is the number of alternative routes. In conclusion, the solving progress for this complex problem is obviously challenging.

As the proposed model is a linear programming, we apply the IBM ILOG CPLEX 12.8 .0 as a computation platform, by adjusting constraints with different scheduling rules in a two-step solving process. Our experiments are all executed on a machine with an Intel (R) Core (TM) i7-7700 processor CPU $3.60 \mathrm{GHz}$ and $8.00 \mathrm{~GB}$ memory. Considering the difficulty in solving process, a two-step solving program with three kinds of scheduling rules are applied, such as space headway, headway time interval, and free-conflict rule. By comparison with the corresponding solution qualities and computational times, headway time interval and free conflicts are adopted in the first step and the further optimization is executed in the second step with scheduling rule related to dynamic space headway (i.e., the core idea comes from the quasi-moving blocking system):

(1) Space headway ( $\mathrm{SH})$ : this strategy is used to control the spatial distance between two consecutive trains according to the real-time speed of the following train, with the ambition to make full use of given capacity. In the other words, the dynamic safety braking distance is considered in the model and constraints (5) (6) are available. This strategy is finally applied in the second step in the solving process.

(2) Headway time interval (HTI): this strategy makes consecutive trains keep the same time interval, without considering the real space interval. In other words, it requires that there are at least $\delta$ time units between occupation times for the same section between two consecutive trains. Consequently, constraints (25) (26) are involved in the model. They are considered in the first step in the solving process:

$$
\begin{aligned}
\omega_{j, q}-\omega_{i, p} \geq & \delta_{p, q}-\left(1-\lambda_{i, j, p, q}\right) M, \\
& \forall i, j \in T,(p, q) \in A, i \neq j, \\
\omega_{i, q}-\omega_{j, p} \geq & \delta_{p, q}-\lambda_{i, j, p, q} M, \\
& \forall i, j \in T,(p, q) \in A, i \neq j .
\end{aligned}
$$

(3) Free-conflict rule (FCR): this strategy refers to ignore the conflicts in the open track area to release the amount of constraints related to train control. To be specific, constraints (5) (6) are not involved in the model. and it is supposed that trains run with full-speed regardless of the minimum safety space headway. In the case of computational time, this strategy is applied in the first step in the solving process.

4.2. Case Description. In this section, we test the proposed model on a timetable considering homogeneous high-speed traffic, where 20 trains with the same maximum speed $(300 \mathrm{~km} / \mathrm{h})$ have different stop patterns in a time span from one hour and a half, shown by Figure 7 . In this figure, the lines with green wave belts are trains' running lines with speed levels, and the green wave belt indicates the full-speed of train on the open track area. The infrastructure is a part of China high-speed rail line with 6 stations and a total of 370 block sections, whose length is about 1360 meters per section operated by a quasi-moving block signalling system. The detail parameters related to train speed level are listed in Table 1, where the running time is running time threshold 


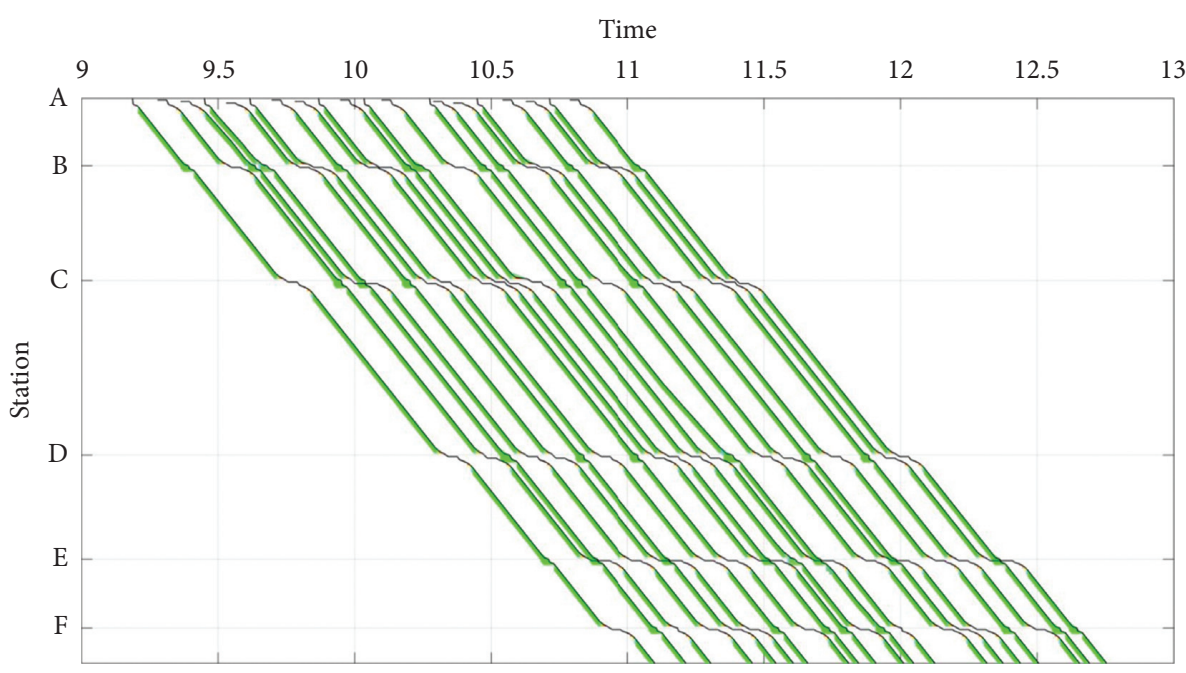

Figure 7: The planned timetable in the normal condition.

Table 1: Train speed level and its running time.

\begin{tabular}{lcc}
\hline Speed level & Train speed $(\mathrm{km} / \mathrm{h})$ & Running time $(\mathrm{s})$ \\
\hline 1 & $\leq 120$ & $16+[24,+\infty)$ \\
2 & $160-120$ & $16+[14,24)$ \\
3 & $200-160$ & $16+[8,14)$ \\
4 & $250-200$ & $16+[3,8)$ \\
5 & $300-250$ & $16+[0,3)$ \\
\hline
\end{tabular}

that the train passed through one block section with the given speed level. And the other parameters of rail line and infrastructure are listed in Figure 8. All the trains are planned to stop at station $\mathrm{C}$ and station D. Primary delays affect all trains' running and follow a 3-parameter Weibull distribution (i.e., $\beta_{h}=1.48, \eta_{h}=560$, and $\gamma_{h}=205$ ). The total primary delay mentioned in this paper is 1019 seconds shown in Figure 8. Furthermore, there are four disturbance cases, which contain two speed limitations and two siding line blockages, occurring simultaneously, and their parameters, i.e., sections impacted, start time, and end time, are demonstrated in Figure 8.

4.3. Computation Time Analysis. The proposed MILP model has a huge number of constraints, growing quadratically with the amount of trains and the amount of sections. And due to the involvement of siding lines adjustment, there are several alternative arrival paths and departure paths for each train at each station. Thus, the involvement of train rerouting makes the NP-hard problem (i.e., train rescheduling) more complicate, and obtaining a fine solution in a limited computational time is hard. Furthermore, high-speed train speed control puts an increasing combinatorial load on the solution process.

Considering the three difficulties in solution computation, we devised an efficient and effective two-step solution process to determine quickly an initial solution by ignoring conflicts in segments in the first step. In other words, the HTI and FCR scheduling rules are applied in the first step, and only conflict detection and resolution in station area are addressed. In order to keep the lower bound feasible, the train order of departure at the previous station $\left(\lambda_{i, j}, p, q\right)$ should consist with the order of arrival at the next station. In the second step, certain values of decision variables, i.e., $z_{t, s}$, $\lambda_{i, j, p, q}, \operatorname{dev}_{t, s}$, and $\mu_{i, j, \eta}$ obtained from the first step are known before computation, while start times of sections in station area $\left(\omega_{t}, s\right)$ obtained in first step are put into the second step. In this way, train arrival/departure paths, train orders, and start times in railway station are fixed before computation in the second step, and then the SP scheduling rule is used in this process. Based on a lot of experimental tests, it is proved that most of cases can be solved within 600 seconds, e.g., $500 \mathrm{~s}$ for first step and $100 \mathrm{~s}$ for the second step.

In order to verify solutions, quality of the solution approach, a benchmark solving approach referred by [18], is considered, where train speed is fixed in the first step and then dynamic space headway is executed secondly. The performances of those two methods are compared through an experimental test, whose parameters are described in Figure 9, but all trains run on time without primary delays. The detailed performance of solutions with computational time is shown in Figure 9, where the solid line denotes the objective value calculated by the proposed approach, while the dash line represents that of the benchmark approach, and red line is about the first step and dark line relates the second step. As a result, the solid curve shows that the objective value is around 8500 when computation time is about 500 seconds, but when computation time is 1700 seconds, the value of dash line responds to 115000 , which is much higher than the value computed by proposed approach, although the computation time taken by benchmark approach is 2 times longer. In a word, the proposed method can get a much better solution within an acceptable time, compared with the benchmark solution approach. The application of HI scheduling rule can accelerate the solving process efficiently. In addition to that, 32 cases with different speed limitations and with/without siding line blockage are tested to study the performance of the solving method 


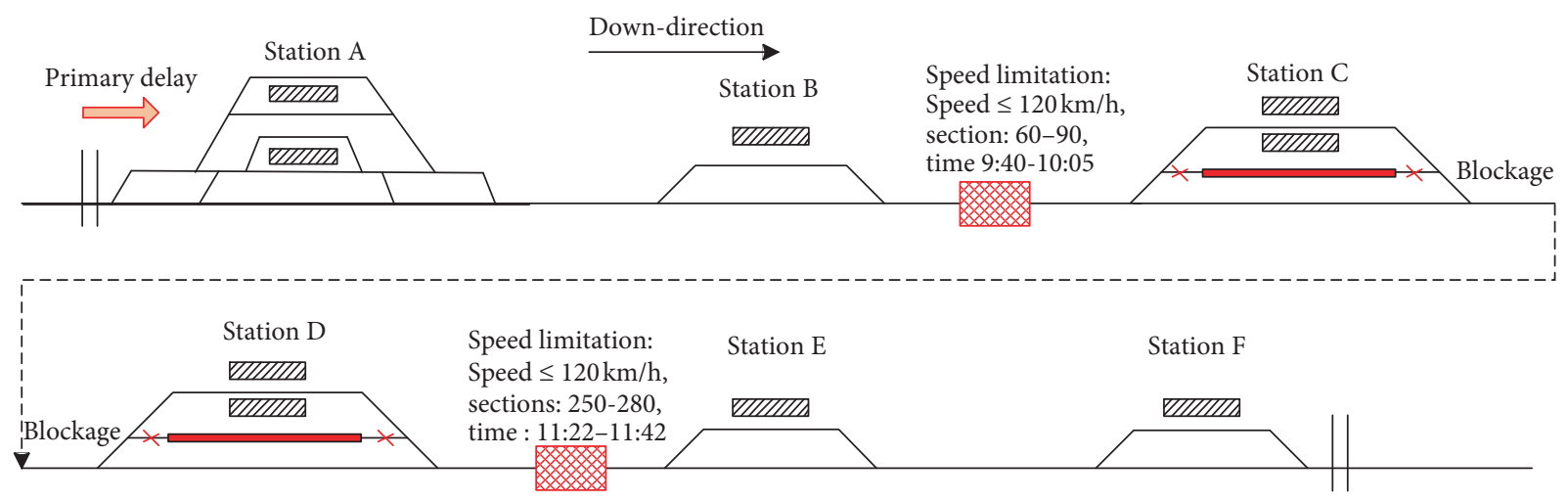

FIGURE 8: Parameters of railway infrastructure and disturbances.

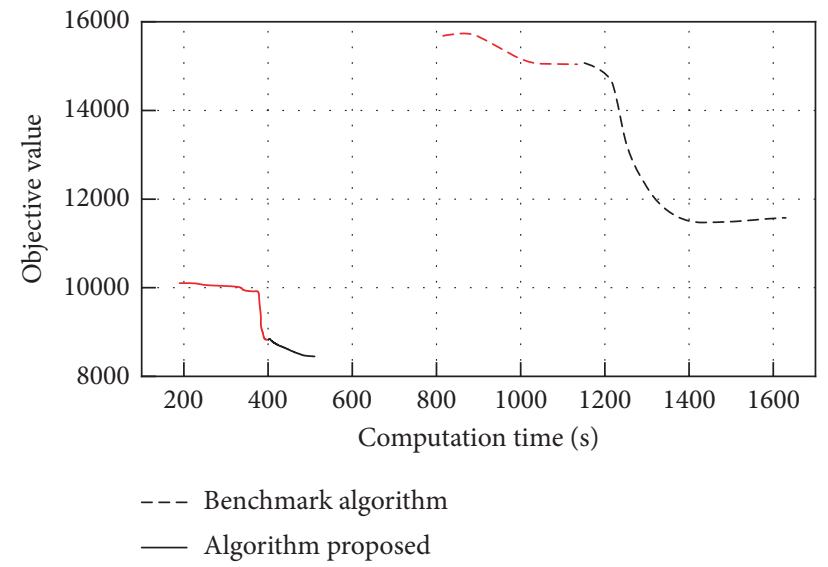

FIGURE 9: Solutions of the proposed and benchmark algorithms with computation times.

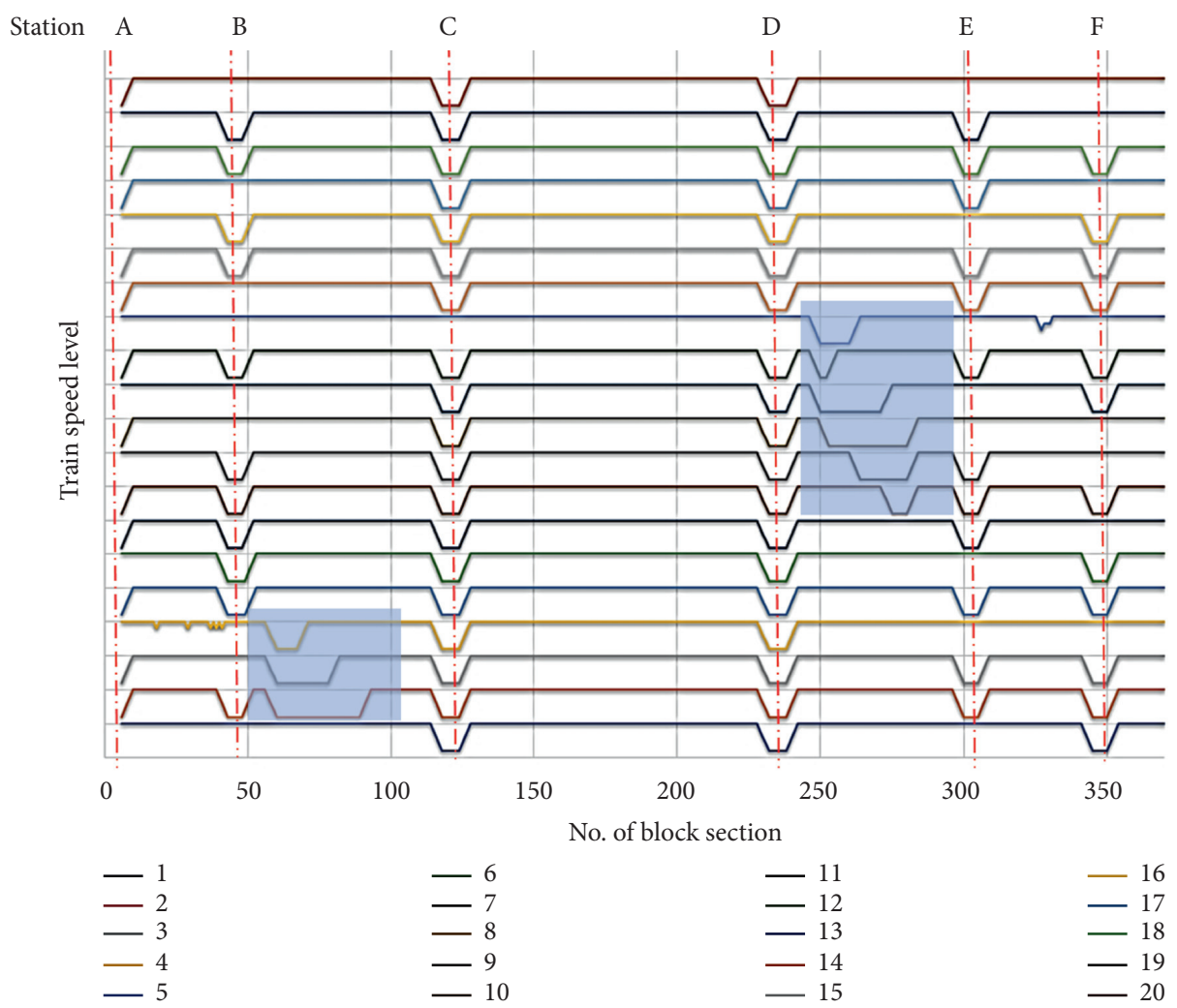

FIgURE 10: Speed levels of 20 trains along their routes. 


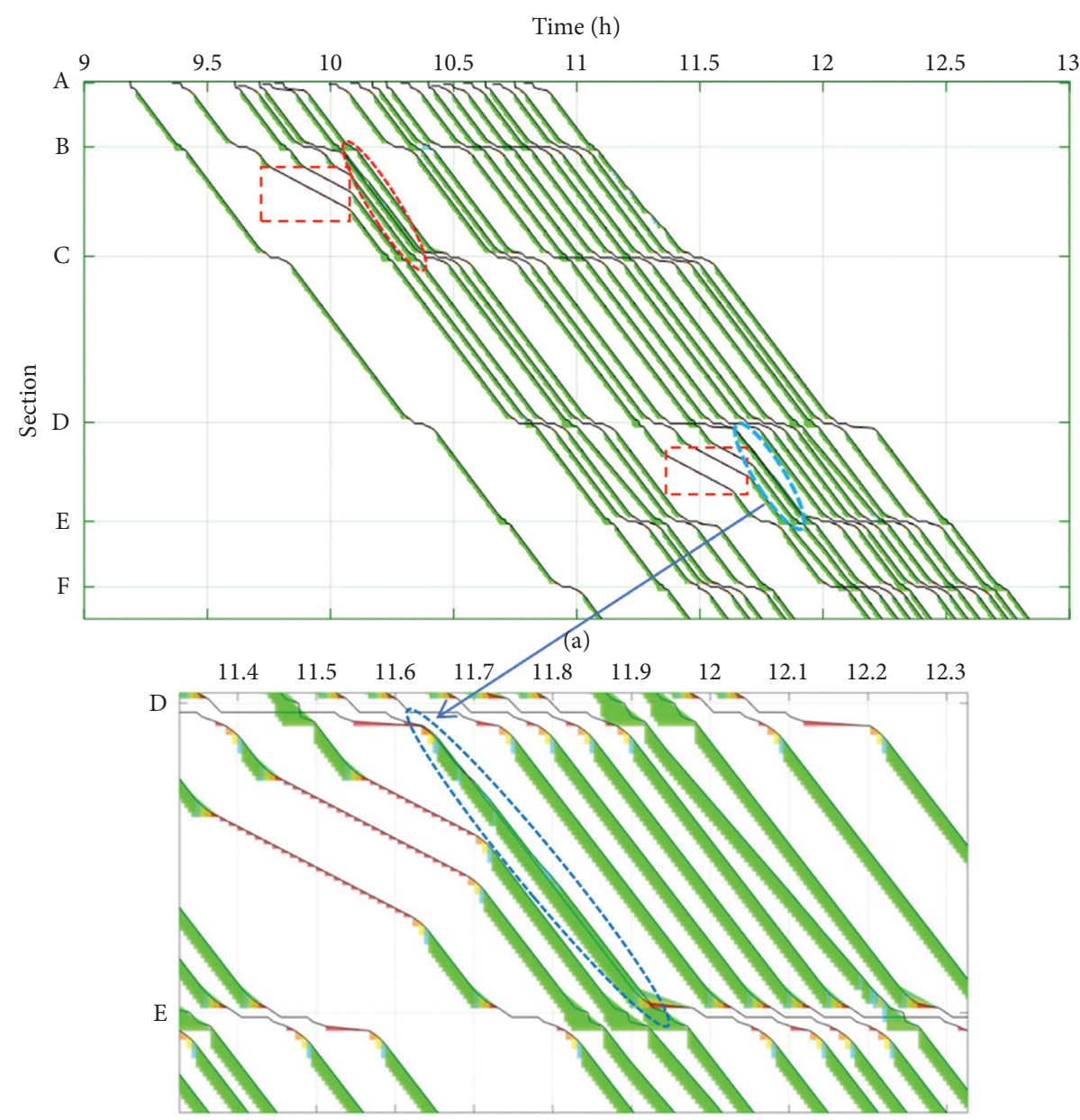

(b)

Figure 11: Rescheduled timetable after the first-step computation.

proposed. If the computation time is 600 seconds, all of them can be solved to get the best or optimal solutions within $5 \%$ optimality gap or less, and it is proved that the setting of disturbances listed in Figure 8 is the most difficult to be solved to get the best solution with $3 \%$ optimality gap under the given computation time.

\section{Results Analysis}

Based on the data listed in Figure 8, solutions solved by the model are analysed in this part. First of all, we analysed the speed level of the given trains, which are plotted in Figure 10. In detail, 20 broken lines represent speed levels (vertical axis) of 20 trains along their routes (horizontal axis). It is noted that the value of vertical axis is cycle number with 5 units divided by each horizontal line. In detail, the start value is speed level 1 and the end is speed level 5 along the upward direction in each cycle period. In terms of undulations of lines in railway station area, trains have to decelerate and accelerate their speed to aboard and alight passengers. Furthermore, the fluctuation (highlighted by the rectangular shaded area) of lines no.2-4 and no.8-13 in the open track area indicated the speed limitation effected by disturbances. In addition to above sections mentioned, all the lines can keep the full-speed on the track sections as most as possible. In this way, it is not only that trains speed are reduced according to the equipment of disturbance of speed limitation, but also the capacity of high-speed railway is maximized by the proposed method.

Regarding to the graphical description for the rescheduled timetable, solutions of the first-step and second-step computation are given in Figure 11 and Figure 12, respectively, where 20 trains' running lines with five kinds of colors denote different speed levels. More precisely, red, purple, yellow, blue, and green colors in running lines correspond to 1, 2, 3, 4, and 5 levels of train speed levels, respectively (i.e., the detail refers Table 1). Furthermore, there are two subpictures in both figures, where the upper figure is the whole rescheduled timetable and the bottom picture presents a partial magnifying timetable denoted by the blue cycle in the up figure. In this figure, there are two obvious parts denoting by two rectangle dotted boxes where trains' speed reduced sharply due to speed limitations between stations B and C or stations D and E. It proved that the proposed model can control the speed of trains accurately to keep the safety of trains along railway track, though there are two speed limitations with different geographical locations and time spans. Secondly, reordering trains and retiming 


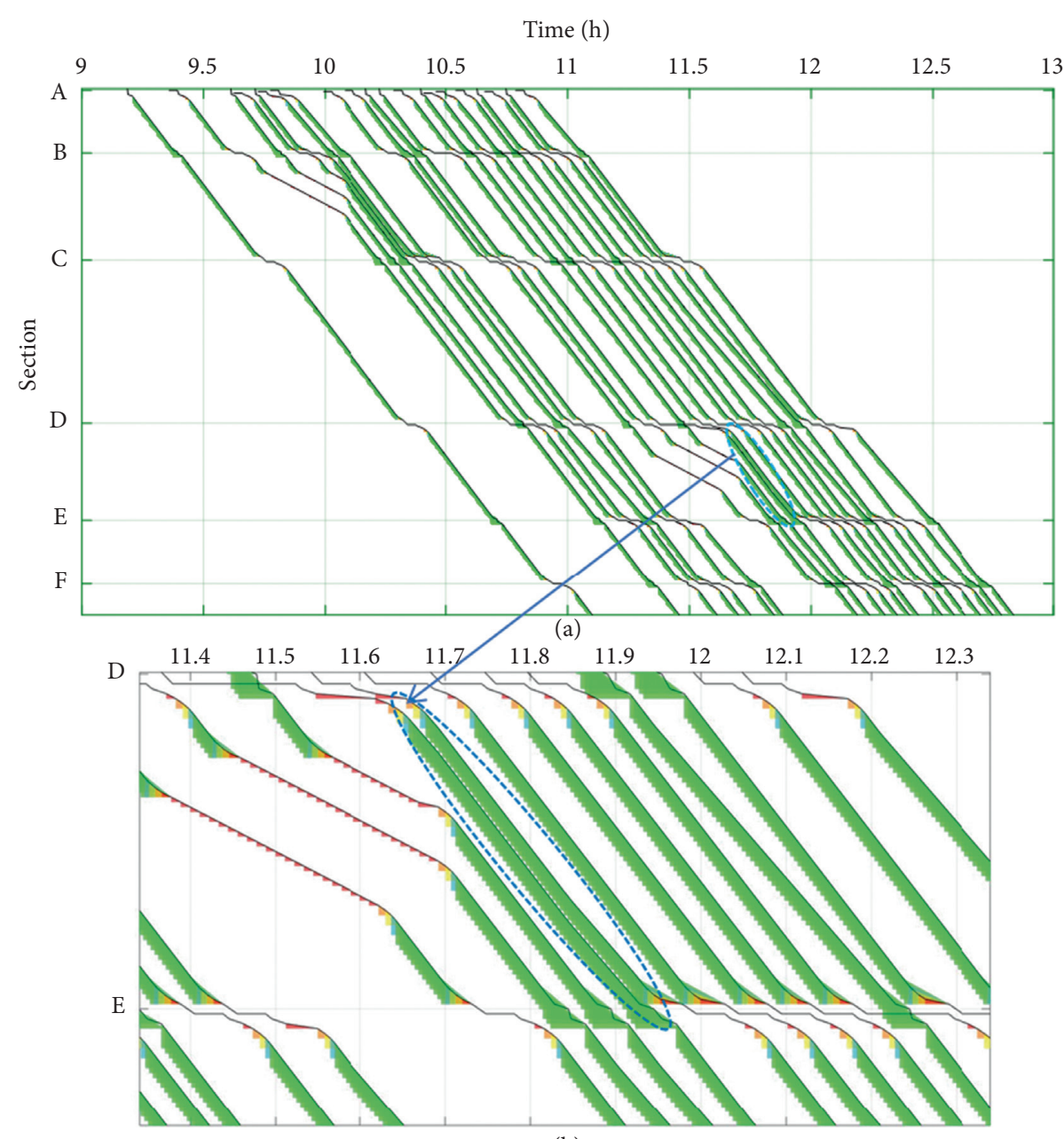

(b)

FIgURE 12: Rescheduled timetables after the second-step computation.

trains are both executed to avoid some potential conflicts, resulting from primary delay or infrastructure capacity.

Comparison with Figures 11 and 12, the arrival or departure times of trains at railway stations are almost the same, but train orders and running times on sections are different, as well as conflicts between trains. To be specific, there are some crosses or overlaps between trains' running lines in the dash circles in Figure 11 and an obvious overlap between trains in the bottom picture in Figure 11, considering the crosses in open track area or overlaps reflect conflicts caused by overpassing of trains in segment. This phenomenon must be forbidden in realistic railway operation, as it means at least two trains occupy the same section at the same time. Nevertheless, those two consecutive trains run on the segment with the maximum speed and minimum headway distance in the bottom picture in Figure 12. As a result, the solution computed by the first-step computation under the control of headway time interval and free-conflict principle is not feasible and reliable sometimes. Given the unreliability of the solution, the further measure should be carried out by the train scheduler or diver, if needed.

With application of the strict headway space rescheduling rule in second-step solution, the running time of each train in each block section and its real-time speed level are coordinate and the final solution is feasible enough. All in all, the proposed mathematical model and the solving method with the two-step algorithm are able to address train rescheduling problem under the influence of the multidisturbances effectively.

Concerning blockages of siding line in station $\mathrm{C}$ and station $\mathrm{D}$, a case without siding line blockage is used as the benchmark. Figure 13 shows negative influence of siding line blockage, especially in arrival path areas in station C. It should be noted that two dash lines in Figure 13 represent the siding lines in the geographical axis. Compared the timetable adjusted with siding line blockage (shown by Figure 13) with that without siding line blockage (shown by Figure 14), it is noticeable that some trains (in Figure 13) have to wait outside station until the former train departure, due to the limited capacity of available siding lines.

Considering the shortage of capacity caused by siding line blockage in railway station, the waiting times of trains in front of station $\mathrm{C}$ related to those two cases are analysed in Figure 15, where each column denotes waiting time of each train, i.e., the blue one relates to Figure 13 and the red one relate to Figure 14. Compared with values of blue columns 


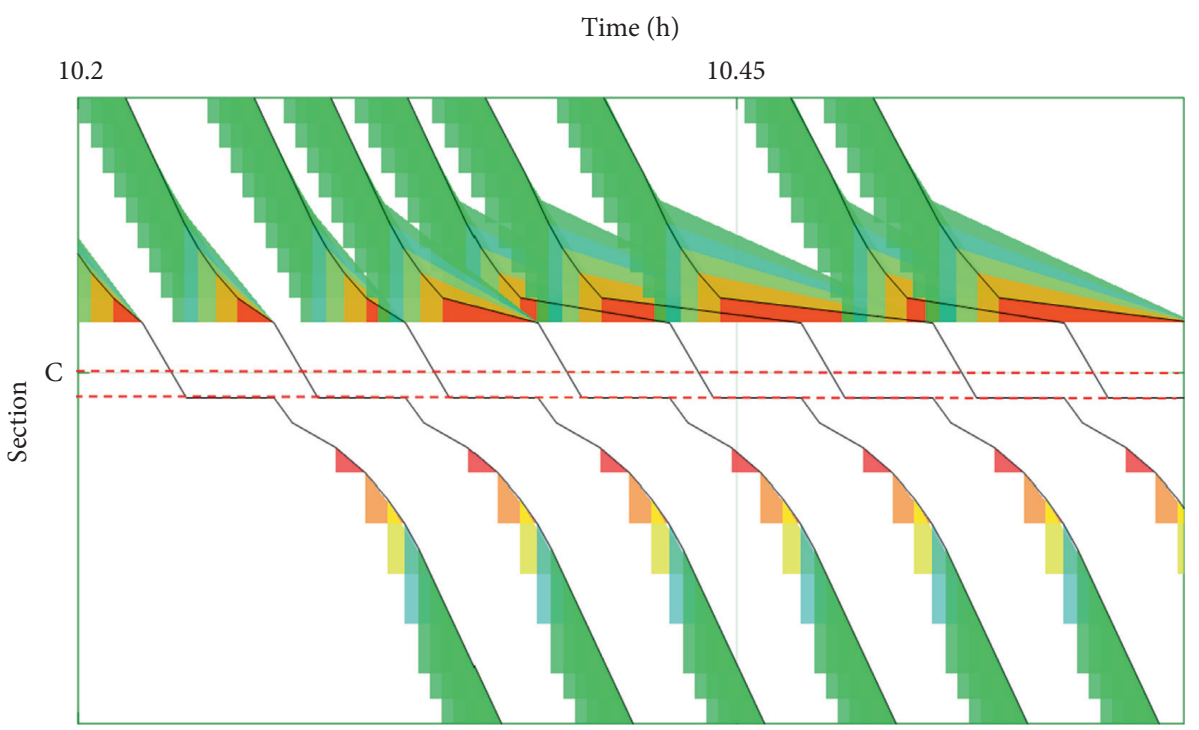

FIGURE 13: Timetable adjusted without siding line blockage in station C.

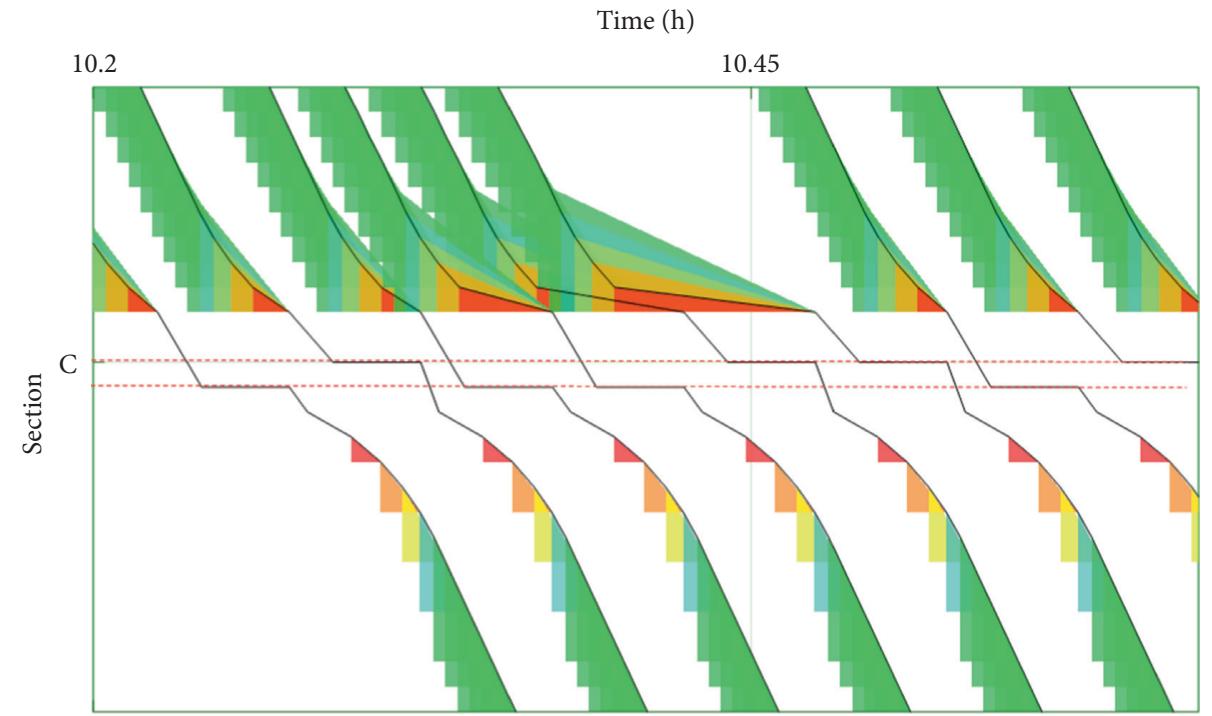

FIgure 14: Timetable adjusted with siding line blockage in station C.

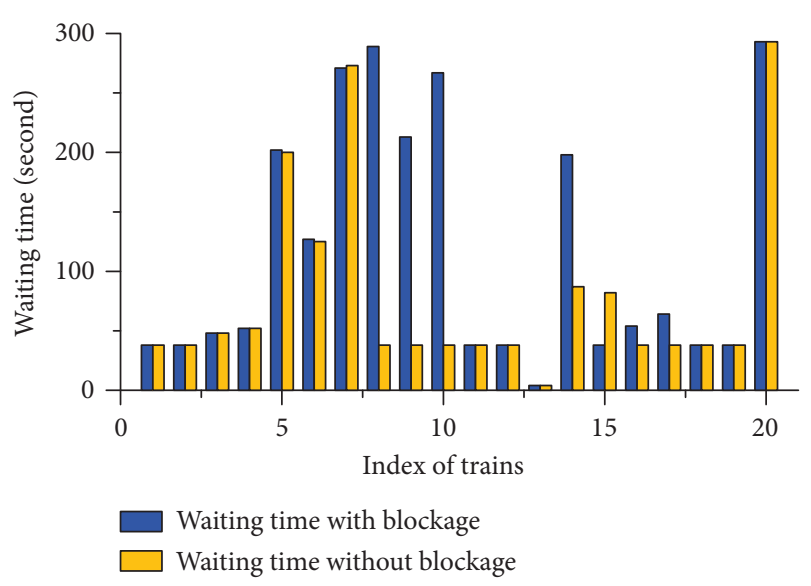

FIgURE 15: Waiting time of each train in front of station C. and yellow columns, some of blue columns are much higher than yellow columns. To be specific, the waiting times of train $8^{\text {th }}, 9^{\text {th }}, 10^{\text {th }}$, and $14^{\text {th }}$ in front of railway station are longer than that without blockage, and around 700 seconds are generated due to blockage of siding line in station C.

\section{Conclusions and Outlook}

Based on above content, the proposed MILP model is able to deal with the problem caused by primary delays, speed limitations, and blockages of siding lines at the same time, and the characteristics of the quasi-moving block signalling system are considered in the model, which make train control possible along with train rescheduling. It succeeds in extending the alternative graph method mentioned by [18] and making rerouting possible along with retiming and 
reordering, although the signalling system of high-speed railway is more flexible and dynamic. Concerning above experimental tests, we draw conclusions as follows:

(1) The proposed MILP model can deal with multidisturbances (i.e., multispeed limitations, primary delay, and siding blockage) in one iteration efficiently, by means of controlling train speed, changing orders of trains, adjusting running times of trains, and resetting arrival/departure paths in railway station area, so it is a comprehensive mathematical optimal method.

(2) The given two-step solution approach can accelerate the solving process by ignoring the conflicts between trains (i.e., overtaking) in segments, in the other words, those constraints related to headway time interval are considered in the railway station area firstly. The comparison with a benchmark algorithm is executed, and the priority of the proposed method is verified.

(3) Timetable adjusted by the mathematical model is feasible and improved, where all conflicts are solved, based on a realistic high-speed railway line in China. Furthermore, all the trains are distributed with reasonable routes and given accurate speed levels from the original to its destination.

There are some shortages remained to improve for future research. First of all, the comparisons with current other methods related to train rescheduling will be under investigation to study the effectiveness and generality of the proposed method. Secondly, there are still a high-speed railway network controlled by a quasi-moving block system is still needed to explore by the proposed method, in terms of dimension of research objective, $\mathrm{N}$-track railway line, and so on. At last, concerning the disadvantage of commercial solving software, a more powerful and efficient heuristic algorithm should be designed and applied to solve the NP-hard problem, in order to meet the requirement of real-world railway operation.

\section{Data Availability}

Some or all data, models, or code that support the findings of this study are available from the corresponding author upon reasonable request.

\section{Conflicts of Interest}

The authors declare that there are no conflicts of interest regarding the publication of this paper.

\section{Acknowledgments}

This work was supported by the National Nature Science Foundation of China (nos. 71801019 and 52072044), the Shannxi Science and Technology Project (nos. 2020JQ-390 and 2020JQ-363), and the Open Project of State Key Laboratory of Traction Power under grant no. TPL2108.

\section{References}

[1] F. Corman and L. Meng, "A review of online dynamic models and algorithms for railway traffic management," IEEE Transactions on Intelligent Transportation Systems, vol. 16, no. 3, pp. 1274-1284, 2015.

[2] G. Cavone, M. Dotoli, N. Epicoco, and C. Seatzu, "A decision making procedure for robust train rescheduling based on Mixed Integer Linear Programming and Data Envelopment Analysis," Applied Mathematical Modelling, vol. 52, pp. 255-273, 2017.

[3] M. Dotoli, N. Epicoco, M. Falagario et al., "A decision support system for real-time rescheduling of railways," in Proceedings of the 13th IEEE European control conference. (ECC 2014), Strasbourg, France, June 2014.

[4] Y. Ye and J. Zhang, "Accident-oriented delay propagation in high-speed railway network," Journal of Transportation Engineering, Part A: Systems, vol. 146, no. 4, Article ID 04020011, 2020.

[5] V. Cacchiani, D. Huisman, M. Kidd et al., "An overview of recovery models and algorithms for real-time railway rescheduling," Transportation Research Part B: Methodological, vol. 63, pp. 15-37, 2014.

[6] T. Dollevoet, D. Huisman, M. Schmidt, and A. Schöbel, "Delay management with rerouting of passengers," Transportation Science, vol. 46, no. 1, pp. 74-89, 2012.

[7] M. Dotoli, N. Epicoco, M. Falagario et al., "A real time traffic management model for regional railway network under disturbances," in Proceedings of the 9th IEEE International Conference on Automation Science and Engineering (CASE 2013), Madison, WIUSA, August 2013.

[8] F. Corman, A. D'Ariano, A. D. Marra, D. Pacciarelli, and M. Samà, "Integrating train scheduling and delay management in real-time railway traffic control," Transportation Research Part E: Logistics and Transportation Review, vol. 105, pp. 213-239, 2017.

[9] W. Fang, S. Yang, and X. Yao, "A survey on problem models and solution approaches to rescheduling in railway networks," IEEE Transactions on Intelligent Transportation Systems, vol. 16, no. 6, pp. 2997-3016, 2015.

[10] I. A. Hansen and J. Pachl, Railway Timetabling \& Operations, Eurailpress, Hamburg, Germany, 2014.

[11] S. Zhan, L. G. Kroon, L. P. Veelenturf, and J. C. Wagenaar, "Real-time high-speed train rescheduling in case of a complete blockage," Transportation Research Part B: Methodological, vol. 78, no. 8, pp. 182-201, 2015.

[12] L. P. Veelenturf, M. P. Kidd, V. Cacchiani et al., "A railway timetable rescheduling approach for handling large-scale disruptions," Transportation Science, vol. 50, no. 3, pp. 841-862, 2015.

[13] A. D'Ariano, D. Pacciarelli, and M. Pranzo, "A branch and bound algorithm for scheduling trains in a railway network," European Journal of Operational Research, vol. 183, no. 2, pp. 643-657, 2007.

[14] F. Corman, A. D’Ariano, D. Pacciarelli, and M. Pranzo, "A tabu search algorithm for rerouting trains during rail operations," Transportation Research Part B: Methodological, vol. 44, no. 1, pp. 175-192, 2010.

[15] M. Samà, A. D'Ariano, F. Corman, and D. Pacciarelli, “A variable neighbourhood search for fast train scheduling and routing during disturbed railway traffic situations," Computers \& Operations Research, vol. 78, no. 2, pp. 480-499, 2017.

[16] M. Samà, C. Meloni, A. D'Ariano, and F. Corman, “A multi-criteria decision support methodology for real-time 
train scheduling," Journal of Rail Transport Planning \& Management, vol. 5, no. 3, pp. 146-162, 2015.

[17] M. Mazzarello and E. Ottaviani, "A traffic management system for real-time traffic optimisation in railways," Transportation Research Part B: Methodological, vol. 41, no. 2, pp. 246-274, 2007.

[18] P. Xu, F. Corman, Q. Peng, and X. Luan, "A train rescheduling model integrating speed management during disruptions of high-speed traffic under a quasi-moving block system," Transportation Research Part B: Methodological, vol. 104, pp. 638-666, 2017.

[19] P. Xu, F. Corman, Q. Peng, and X. Luan, "A timetable rescheduling approach and transition phases for high-speed railway traffic during disruptions," Transportation Research Record: Journal of the Transportation Research Board, vol. 2607, no. 1, pp. 82-92, 2017.

[20] S. Long, L. Meng, Y. Wang et al., "A train rescheduling optimization model with considering the train control for a high-speed railway line under temporary speed restriction," in Proceedings of the 2019 IEEE Intelligent Transportation Systems Conference (ITSC), pp. 2809-2816, Auckland, New Zealand, October2019.

[21] X. Li, B. Shou, and D. Ralescu, "Train rescheduling with stochastic recovery time: a new track-backup approach," IEEE Transactions on Systems, Man, and Cybernetics: Systems, vol. 44, no. 9, pp. 1216-1233, 2014. 